\title{
Efficiency Analysis of a Ladder Multilevel Converter with the Use of the Equivalent Continuous Model
}

\author{
Andrés Lopez ${ }^{*}$, Diego Patino ${ }^{\dagger}$, and Rafael Diez ${ }^{*}$ \\ ${ }^{*}$ Department of Electronics, Pontificia Universidad Javeriana, Bogotá, Colombia
}

\begin{abstract}
This study analyzes a ladder multilevel converter (double ladder topology) with the use of a new averaging modeling technique. This technique introduces an analytical method to compute for the switching losses and is used to conduct an in-depth analysis of the influence of the switching frequency and parasitic resistance of components on converter efficiency. The obtained results enable the selection of switches and switching frequency to minimize losses. Moreover, simulation results and experimental measurements validate the analytical calculations.
\end{abstract}

Key words: Average modeling, Ladder multilevel converter, Power losses

\section{INTRODUCTION}

Achieving high efficiency on power converters is one of the main issues of power electronics [1], [2], [37], [38]. Two main issues related to efficiency are conduction and switching losses. Conduction losses can be resolved by reducing the ON resistance on power MOSFETs, reducing the forward voltage on power diodes or IGBTs and using passive components with low parasitic resistance [22]-[26]. Semiconductor devices are also becoming faster. Power diodes with low reverse recovery current have also been developed to reduce switching losses [3].

In addition to the reduction of switching losses, semiconductor devices with high reverse voltage have also been developed. Although these new devices expand the possibilities for high-voltage and high-frequency use, classical converters, such as buck or boost, still face some limitations in terms of applications of several kilovolts [4]-[8], [27], [28]. Multilevel converters were proposed to deal with the high-voltage problem of classical converters. Multilevel converters manage the high-voltage problem (several kilovolts) with the use of low-voltage components (hundreds of volts). Among the multilevel converters, some, such as the ladder multilevel DC/DC converter, use only capacitive components

Manuscript received May 10, 2014; accepted Oct. 4, 2014

Recommended for publication by Associate Editor Kyo-Beum Lee.

†Corresponding Author: patino-d@javeriana.edu.co

Tel: +57-3208380 Ext. 5550, Pontificia Universidad Javeriana

*Dept. of Electronics, Pontificia Universidad Javeriana, Colombia (switched capacitors (SC)) in their power circuit, reducing size and weight [9]-[15], [29]-[31]. In [16], the classical ladder topology (CLT) and two variations were presented. The double ladder topology (DLT) was shown to produce less output resistance and less output voltage ripple, which makes this topology highly efficient and suitable for implementation.

In addition to efficiency, a control law is required by power converters. In most cases, a mathematical model of the converter is required. These converters are often modeled with the use of the classical averaging technique [32]. However, this technique works under the assumption of slow dynamics on each switching state. If such assumption is unfulfilled, then an inaccurate model can be obtained.

In an SC converter, capacitors with different voltages can be connected in parallel, producing current spikes. Given that the equivalent series resistance (ESR) of the capacitors and the ON resistance of the interconnection switches are usually small, the equivalent time constant $\tau$ of the resulting circuit is also small. Therefore, the dynamics are rapid compared with the switching period $T$ (see Fig. 1). With consideration of the rapid dynamics of the current of the capacitors, the assumption for the classical averaging model is unfulfilled. In [17], a new analytical modeling technique that obtains an equivalent continuous model of a switched converter regardless of the dynamics on each switching state is proposed. The technique is called generalized equivalent continuous model (GECM). In this study, this technique is used to analyze the behavior of the converter and optimize its efficiency.

Fig. 1 shows that, for a small $\tau$ (i.e., small ESR and switch 


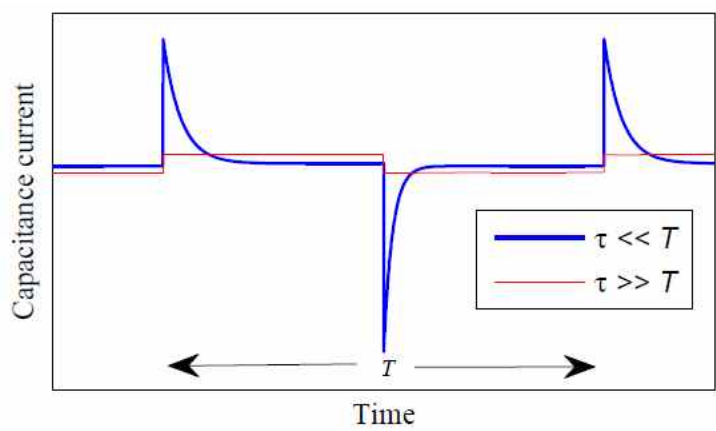

Fig. 1. Capacitor current waveform for different $\tau$ values. $T$ represents the switching period.

ON resistance), the current waveform presents an exponential shape and higher current spikes than for a large $\tau$ value (i.e., higher ESR and switch ON resistance). Moreover, given the charge balance, the waveforms should have the same average in each cycle. If the average is the same, then we can conclude that the exponential waveform has a higher root mean square (RMS) value. Therefore, reducing the resistor value $R$ implies an increase in the $R M S$ value. Given that the power losses in resistor $R$ are expressed as $i_{R M S}^{2} R$, no conclusion on power losses can be easily drawn.

In [1], [18]-[20], [33], and [34], power losses in SC converters are resolved and analyzed. In SC converters, reducing the $\mathrm{ON}$ resistance of the switches or ESR does not necessarily imply higher efficiency. In [1] and [18], analytical calculations of the conduction energy losses of an SC converter are presented. These calculations are essentially the same that the GECM conducted under all matrix operations. Therefore, the results of conduction losses only are the same. However, the GECM is easier to compute using a convenient state space form and works for any circuit topology modeling its dynamics. In [33], an analysis method that completely omits parasitic resistances is presented. Therefore, it cannot be computed in the state space form (GECM). References [20] and [34] provide a basic mathematical demonstration on how an ideal SC converter (without parasitic resistances) generates energy losses and how to prevent energy losses. However, no specific calculation of the losses is conducted. In [19], a different approach is presented. Using the example proposed in Section VI of reference [19], an approximation of the output resistance is obtained at the switching frequency of $30 \mathrm{MHz}$, producing a $5 \%$ error. This error can be eliminated using GECM. Notably, none of the previous works considered switching losses.

In this study, the GECM approach is applied to the DLT converter with eight cells for high-voltage application. The components of the converter are selected to optimize its efficiency with consideration of conduction and switching losses. Moreover, the converter is implemented and experimental measurements are taken.

This paper is organized as follows: Section II presents the DLT. Section III highlights the different modeling techniques.

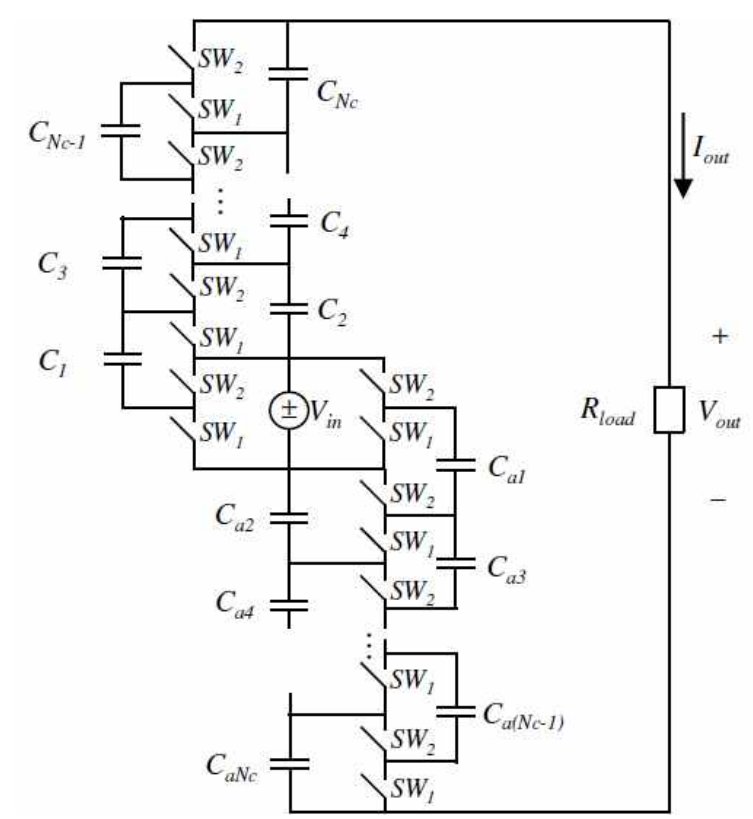

Fig. 2. DLT of a DC/DC voltage multilevel elevator with $N_{C}$ cells. $S W_{1}$ and $S W_{2}$ are complementary. Average currents are shown during each cycle.

Section IV analyzes the switching losses. Finally, Section V demonstrates the efficiency of the DLT with the use of the new modeling technique.

\section{LADDER MUltileVEL CONVERTER}

Fig. 2 shows the generalized DLT proposed in [16]. This converter has $N_{C}$ basic cells (Fig. 3) and an ideal output voltage $V_{\text {out }}=\left(N_{C}+1\right) V_{\boldsymbol{i}}$. This converter works with two control signals, one with $50 \%$ duty cycle for switches $S W_{1}$ and the other complementary for switches $S W_{2}$ [21], [35]. The DLT presents less output voltage drop and less ripple in comparison with the CLT [16].

The basic cell shown in Fig. 3 consists of two capacitive elements $\left(C_{1}\right.$ and $\left.C_{2}\right)$ and four switching components (two $S W_{1}$ and two $\left.S W_{2}\right)$. When switches $S W_{1}$ are ON while $S W_{2}$ are $\mathrm{OFF}$, the input voltage source $V_{\boldsymbol{i}}$ charges the capacitor $C_{1}$. In the other half cycle, with $S W_{2} O N$ and $S W_{1}$ OFF, the capacitor $C_{1}$ charges the capacitor $C_{2}$, which ideally results in $V_{\text {out }}=2 V_{\boldsymbol{i}}$.

The connection between capacitors is affected by the ON resistance of the switches and the ESR of each capacitor, thus producing voltage drops. Furthermore, the time constant of the equivalent circuit, which depends on the capacitance and resistance values, affects the current waveform on the capacitors: a large time constant produces a square waveform, whereas a small time constant produces an exponential waveform, as previously shown in Fig. 1.

As stated previously, when the time constant is small compared with the switching period, the classical averaging model cannot produce an accurate model [17]. 


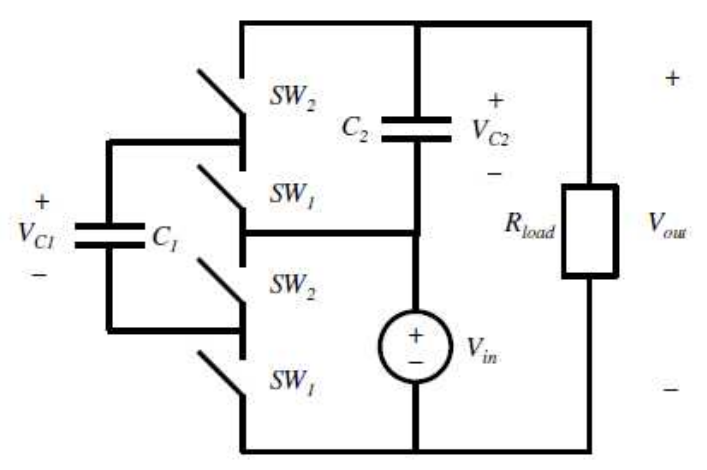

Fig. 3. Basic cell of the ladder multilevel converter.

\section{MOdELING TECHNIQUES}

The model of ladder converters can be written as a switched system. Mode 1 is represented by $\sigma=1$ and occurs when $S W_{1}=\mathrm{ON}\left(S W_{2}=\mathrm{OFF}\right)$. Mode 2 is represented by $\sigma=2$ and occurs when $S W_{1}=\mathrm{OFF}\left(S W_{2}=\mathrm{ON}\right)$. Thus, the dynamics of the converter can be written as follows:

$$
\begin{aligned}
& \dot{x}= \begin{cases}A_{1} x+B_{1} u & \text { if Mode 1 } \\
A_{2} x+B_{2} u & \text { if Mode 2' }\end{cases} \\
& y= \begin{cases}F_{1} x+G_{1} u & \text { if Mode 1 } \\
F_{2} x+G_{2} u & \text { if Mode 2' }\end{cases}
\end{aligned}
$$

where $x$ and $\dot{x}$ are the voltages of the capacitors and their derivatives, respectively. $u$ represents input variables (input voltage $V_{\dot{n}}$ ) and $y$ represents output variables (output voltage $V_{\text {out }}$ ). The matrices of each mode $A_{1}, B_{1}, F_{1}, G_{1}$ and $A_{2}, B_{2}, F_{2}, G_{2}$ can be computed using classical circuit analysis. The values of these matrices depend on the value of each capacitor, as well as the values of the ESR and the resistances of the switches.

From this switched model, we can deduce two modeling techniques, namely, the classical averaging model and the GECM.

\section{A. Classical Averaging Model}

When we assumed that the state space vector $x$ is constant when each mode is active, the classical averaging technique is deduced [32]. With this assumption, the average model from Eqs. (1) and (2) can be computed as follows:

$$
\begin{gathered}
\langle\dot{x}\rangle=\underbrace{\left(A_{1} d+A_{2}(1-d)\right)}_{\langle A\rangle}\langle x\rangle+\underbrace{\left(B_{1} d+B_{2}(1-d)\right)}_{\langle B\rangle}, \\
\langle y\rangle=\underbrace{\left(F_{1} d+F_{2}(1-d)\right)}_{\langle F\rangle}\langle x\rangle+\underbrace{\left(G_{1} d+G_{2}(1-d)\right)}_{\langle G\rangle} u,
\end{gathered}
$$

where $\langle *\rangle$ is the average of the argument and $d$ is the duty cycle. However, as shown in Fig. 1, capacitor voltages in ladder topologies can considerably vary during each mode. Then, classical averaging model is not an appropriate method to characterize ladder converters.

\section{B. Generalized Equivalent Continuous Model}

A new analytical modeling technique was proposed in [17] to deal with high-speed dynamics. In this method, the assumption of slow variation in the state vector $x$ during each mode is eliminated. This subsection briefly presents the method. The following steps show the procedure to obtain this model:

1) Obtain the State Space Matrices of Each Switching State: The analysis starts with the matrices $A_{\sigma}, B_{\sigma}, F_{\sigma}$, and $G_{\sigma}$ for $\sigma=\{1,2\}$. These matrices are obtained from the model shown in Eqs. (1) and (2).

2) Reorganize the State Space Matrices: Previous matrices are unsuitable for the model. Then, the matrices $\mathcal{A}_{\sigma}, \mathcal{F}_{\sigma}, \mathcal{A}_{\sigma}$, and $\mathcal{F}_{\sigma}$ are computed as follows:

$$
\mathcal{A}_{\sigma}=\left[\begin{array}{ll}
A_{\sigma} & B_{\sigma} \\
0 & 0
\end{array}\right] \quad \mathcal{F}_{\sigma}=\left[\begin{array}{ll}
F_{\sigma} & G_{\sigma}
\end{array}\right]
$$

with $\sigma=\{1,2\}$. Thus, the system is transformed into

$$
\begin{gathered}
\dot{z}=\mathcal{A}_{\sigma} Z \\
y=\mathcal{F}_{\sigma} Z
\end{gathered}
$$

where

$$
z=\left[\begin{array}{ll}
x^{T} & u^{T}
\end{array}\right]^{T}
$$

3) Calculate $\phi_{1}(d T)$ and $\phi_{2}((1-d) T)$ : The state transition matrices are computed, but have to be evaluated in $d T$. Thus, $\phi_{1}(d T)$ and $\phi_{2}(d T)$ are expressed as follows:

$$
\phi_{1}(d T)=e^{\mathcal{A}_{1} d T} \quad \phi_{2}(d T)=e^{\mathcal{A}_{2} d T} .
$$

4) Calculate $\mathcal{A}_{\delta}$ : Matrix $\mathcal{A}_{\delta}$ represents the transition matrix in one switching period $T$. The two modes are presented in this matrix. $\mathcal{A}_{\delta}$ is computed as follows:

$$
\mathcal{A}_{\delta}=\phi_{2}((1-d) T) \phi_{1}(d T),
$$

5) Calculate $\Omega_{1}$ and $\Omega_{2}: \Omega_{1}$ and $\Omega_{2}$ are defined as the integrals of the functions $\phi_{1}(t)$ and $\phi_{2}(t)$, respectively. These integrals enable the generation of the average dynamics of each mode, which are obtained as follows:

$$
\Omega_{1}=\frac{1}{T} \int_{0}^{d T} \phi_{1}(\tau) \mathrm{d} \tau \quad \Omega_{2}=\frac{1}{T} \int_{0}^{(1-d) T} \phi_{2}(\tau) \mathrm{d} \tau .
$$

An analytical solution of $\Omega_{1}$ and $\Omega_{2}$ exists when $\mathcal{A}_{\sigma}$, $\sigma=\{1,2\}$ is nonsingular. In this case, we obtain the following equation:

$$
\Omega_{\sigma}=\frac{1}{T} \int_{0}^{d_{\sigma} T} \phi_{\sigma}(\tau) \mathrm{d} \tau=\frac{1}{T} \mathcal{A}_{\sigma}^{-1}\left(\phi_{\sigma}\left(d_{\sigma} T\right)-I\right),
$$

where $d_{1}=d$ and $d_{2}=1-d$. When $\mathcal{A}_{\sigma}$ is singular, it should be numerically computed. Indeed, numerical integration is an approximation of the integral real value.

6) Calculate $\Gamma$ : Defining the matrix $\Gamma$ in the function of $\Omega_{1}$ and $\Omega_{2}$ is also necessary. Using the results of the previous step, the value of $\Gamma$ can be computed as follows:

$$
\Gamma=\Omega_{1}+\Omega_{2} \phi_{1}(d T) .
$$

7) Obtain the Matrix $\langle\mathcal{A}\rangle$ : The proposed continuous model is expressed as follows:

$$
\langle\dot{x}\rangle=\langle A\rangle\langle x\rangle+\langle B\rangle u
$$

Information on $\langle A\rangle$ and $\langle B\rangle$ is inside matrix $\langle\mathcal{A}\rangle$. This matrix describes the average dynamics and can be obtained as follows: 


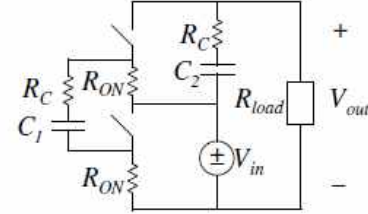

(a)

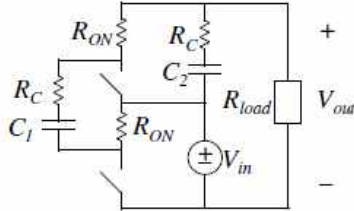

(b)
Fig. 4. Switching modes with parasitic resistances. (a) $S W_{1} \mathrm{ON}$; $S W_{2}$ OFF. (b) $S W_{1}$ OFF; $S W_{2}$ ON.

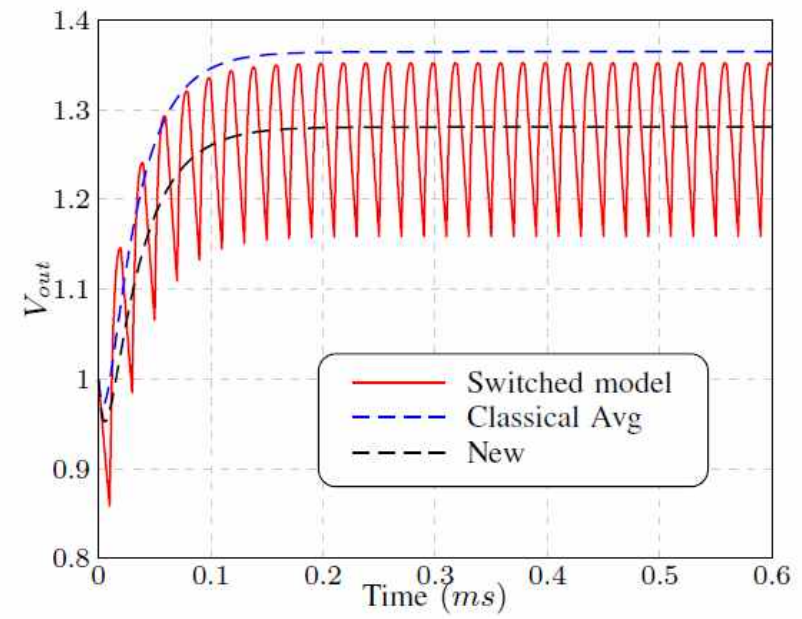

Fig. 5. Comparison of the classical averaging model, the GECM, and the switched response.

$$
\langle\mathcal{A}\rangle=\Gamma\left(\frac{1}{T} \ln \left(\mathcal{A}_{\delta}\right)\right) \Gamma^{-1} .
$$

$\Gamma$ and $\mathcal{A}_{\delta}$ are previously defined. Indeed, $\langle\mathcal{A}\rangle$ has the following form:

$$
\langle\mathcal{A}\rangle=\left[\begin{array}{ll}
\langle A\rangle & \langle B\rangle \\
0 & 0
\end{array}\right] .
$$

Then, $\langle A\rangle$ and $\langle B\rangle$ can be derived from $\langle\mathcal{A}\rangle$.

8) Calculate $\Gamma_{\mathcal{F}}$ : Matrix $\Gamma_{\mathcal{F}}$ is necessary to compute the output model, as follows:

$$
\Gamma_{\mathcal{F}}=\mathcal{F}_{1} \Omega_{1}+\mathcal{F}_{2} \Omega_{2} \phi_{1}(d T) .
$$

9) Obtain the Matrix $\langle\mathcal{F}\rangle$ : The continuous model for $\langle x\rangle$ is obtained using Eq. (14). The continuous model for the output $y$ is obtained as follows:

$$
\langle y\rangle=\langle F\rangle\langle x\rangle+\langle G\rangle u
$$

As shown in Step 7, matrix $\langle\mathcal{F}\rangle$ exists, which contains $\langle F\rangle$ and $\langle G\rangle$. This matrix is expressed as follows:

$$
\langle\mathcal{F}\rangle=\left[\begin{array}{ll}
\langle F\rangle & \langle G\rangle
\end{array}\right],
$$

where $\langle\mathcal{F}\rangle$ is derived from:

$$
\langle\mathcal{F}\rangle=\Gamma_{\mathcal{F}} \Gamma^{-1},
$$

where $\Gamma_{\mathcal{F}}$ and $\Gamma$ are derived from Eqs. (17) and (13), respectively.

10) Obtain the Final State Space Matrices: Finally, the results from Steps 7 and 9 are separated as shown respectively in Eqs. (16) and (19), to obtain the state space matrices $\langle A\rangle,\langle B\rangle,\langle F\rangle$, and $\langle G\rangle$. These matrices describe the average system behavior.

\section{Basic Cell Example}

In this section, the GECM is applied to the basic ladder multilevel converter shown in Fig. 3. Fig. 4 shows the two possible modes of the basic cell, with the parasitic resistances of each component (ESR of capacitors denoted as $R_{C}$ and ON resistance of the MOSFETs denoted as $R_{O N}$ ).

To obtain a model of the system in state space variables, such as in Eqs. (1) and (2), the state space vector $x$, the input $u$, and the output $y$ are defined as follows:

$$
x=\left[\begin{array}{ll}
V_{C_{1}} & V_{C_{2}}
\end{array}\right]^{T}, \quad u=V_{\dot{n}}, \quad y=V_{\text {out }} .
$$

For the mode shown in Fig. 4(a), the state space matrices are calculated as follows:

$$
\begin{aligned}
& A_{1}=\left[\begin{array}{ll}
\frac{-1}{\left(2 R_{O N}+R_{C}\right) C_{1}} & 0 \\
0 & \frac{-1}{\left(R_{C}+R_{\text {bad }}\right) C_{2}}
\end{array}\right], \\
& B_{1}=\left[\begin{array}{ll}
\frac{1}{\left(2 R_{O N}+R_{C}\right) C_{1}} \\
\frac{-1}{\left(R_{C}+R_{\text {bad }}\right) C_{2}}
\end{array}\right], \quad F_{1}=\left[\begin{array}{ll}
0 & \frac{R_{\text {bad }}}{R_{C}+R_{\text {bad }}}
\end{array}\right], \\
& G_{1}=\frac{R_{\text {bad }}}{R_{C}+R_{\text {bad }}} .
\end{aligned}
$$

For the mode shown in Fig. 4(b), the state space matrices are calculated as follows:

$$
\begin{aligned}
& A_{2}=\varpi\left[\begin{array}{ll}
\frac{-\left(R_{C}+R_{b a d}\right)}{C_{1}} & \frac{R_{b a d}}{C_{1}} \\
\frac{R_{b a d}}{C_{2}} & \frac{-\left(R_{C}+R_{b a d}+2 R_{O N}\right)}{C_{2}}
\end{array}\right], \\
& B_{2}=\varpi\left[\begin{array}{l}
\frac{-R_{C}}{C_{1}} \\
\frac{-\left(R_{C}+2 R_{O N}\right)}{C_{2}}
\end{array}\right] \text {, } \\
& F_{2}=\varpi\left[R_{C} R_{\text {bad }} \quad R_{\text {bad }}\left(R_{C}+2 R_{O N}\right)\right] \text {, } \\
& G_{2}=2 \varpi R_{\text {bad }}\left(R_{C}+R_{O N}\right) \text {, }
\end{aligned}
$$

with:

$$
\varpi=\frac{1}{R_{C}\left(R_{C}+2\left(R_{b a d}+R_{O N}\right)\right)+2 R_{b a d} R_{O N}} .
$$

For this numerical example, we considered the following values of the components: $R_{O N}=1.8 \Omega, C_{1}=C_{2}=2.2 \mu \mathrm{F}$, $R_{C}=2.5 \mathrm{~m} \Omega$, and $R_{\text {bad }}=50 \Omega$. The switching frequency for this example is $f=50 \mathrm{kHz}$, with a duty cycle $d=0.5$.

The equivalent state space matrices that describe the system behavior using classical averaging model are computed. The matrices are expressed as follows:

$$
\begin{aligned}
& \langle A\rangle=\left[\begin{array}{ll}
-126.13 & 63.04 \\
63.04 & -78.18
\end{array}\right] \times 10^{3},\langle B\rangle=\left[\begin{array}{l}
63.08 \\
-15.15
\end{array}\right] \times 10^{3} . \\
& \langle F\rangle=\left[\begin{array}{ll}
0.00035 & 0.999
\end{array}\right] \quad, \quad\langle G\rangle=0.999
\end{aligned}
$$

The matrices for the GECM, following the steps previously explained, are expressed as follows:

$$
\begin{aligned}
& \langle A\rangle=\left[\begin{array}{ll}
-134.67 & 65.6 \\
58.78 & -69.65
\end{array}\right] \times 10^{3},\langle B\rangle=\left[\begin{array}{l}
69.81 \\
-19.49
\end{array}\right] \times 10^{3} . \\
& \langle F\rangle=\left[\begin{array}{ll}
0.00011 & 0.999
\end{array}\right] \quad, \quad\langle G\rangle=1
\end{aligned}
$$

Fig. 5 shows a comparison of the step response for both models (classical averaging and GECM) and the real behavior of the converter in simulation. We observed that the GECM produces better results than the classical averaging model because it is capable of computing the real average of $V_{\text {out }}$. 
For more information on this example, see [36].

\section{SWITCHING LOSSES}

In this section, an analytical method to estimate the switching losses of the converter is presented. This method uses the results of the GECM to analyze the system dynamics.

\section{A. General Case}

Let the voltages of the switches (open) and their currents (closed) be organized as follows:

$$
\begin{aligned}
& v_{Q}^{\text {open }}=\left[v_{Q_{1}}^{\text {open }}, v_{Q_{2}}^{\text {open }}, \ldots, v_{Q_{j}}^{\text {open }}\right]^{T}, \\
& i_{Q}^{\text {cbse }}=\left[i_{Q_{1}}^{\text {dbse }}, i_{Q_{2}}^{\text {cbse }}, \ldots, i_{Q_{j}}^{\text {dbse }}\right]^{T},
\end{aligned}
$$

where $j$ is the number of switches.

The matrix $A_{\delta}$ from Eq. (10) defines a discrete model of the initial conditions of the mode $\sigma=1$. Therefore, the following model can be defined using Eq. (26):

$$
\begin{aligned}
z_{0}(T(k+1)) & =\mathcal{A}_{\delta} z_{0}(T k), \\
v_{Q}^{\text {open }} & =\mathcal{H} z_{0}(T k), \\
i_{Q}^{\text {cbse }} & =\mathcal{S} z_{0}(T k),
\end{aligned}
$$

where:

$$
z_{0}=\left[\begin{array}{ll}
x_{0}^{T} & u^{T}
\end{array}\right]^{T},
$$

with $z_{0}$ being the discrete version of $z$ and $x_{0}$ being the discrete version of $x$.

Matrices $\mathcal{H}$ and $\mathcal{S}$ represent the relationship between open voltages $v_{Q}^{\text {open }}$ and closed currents $i_{Q}^{\text {dose }}$ of the switches, the capacitor voltages, and the input $\left(z_{0}\right)$.

Fig. 6 shows the switching waveforms of a switch with a trapezoidal approximation of the switching losses. We assumed that the turn-on and turn-off times are small as compared with the system time constant. Under this assumption, the value of the state space vector is the same right before switching and right after switching (i.e., the value of the state space vector is $z_{0}(0)$ in time instants marked as $a$ and $b$ in Fig. 6). Therefore, the voltages of the switches (open) and their currents (closed) can be computed in terms of $z_{0}$ regardless their state in each subsystem.

Notably, all voltages and currents in the instants marked as $a$ and $b$ in Fig. 6 are calculated. Computing the same voltages and currents in the next switching instant $z_{1}$ is also necessary. The state space vector in the next switching instant can be defined as follows:

$$
z_{1}=\phi_{1}(d T) z_{0}
$$

Using this definition, additional voltages and currents can be computed and added to the system in Eq. (27), expanding matrices $\mathcal{H}$ and $\mathcal{S}$ as follows:

$$
\begin{aligned}
\mathcal{H}^{*} & =\left[\begin{array}{ll}
\mathcal{H}^{T} & \left(\mathcal{H} \phi_{1}(d T)\right)^{T}
\end{array}\right]^{T}, \\
\mathcal{S}^{*} & =\left[\begin{array}{ll}
\mathcal{S}^{T} & \left(\mathcal{S} \phi_{1}(d T)\right)^{T}
\end{array}\right]^{T},
\end{aligned}
$$

Therefore, the new system is defined as follows:

$$
\begin{aligned}
z_{0}(T(k+1)) & =\mathcal{A}_{\delta} z_{0}(T k), \\
v_{Q^{*}}^{\text {open }} & =\mathcal{H}^{*} z_{0}(T k),
\end{aligned}
$$

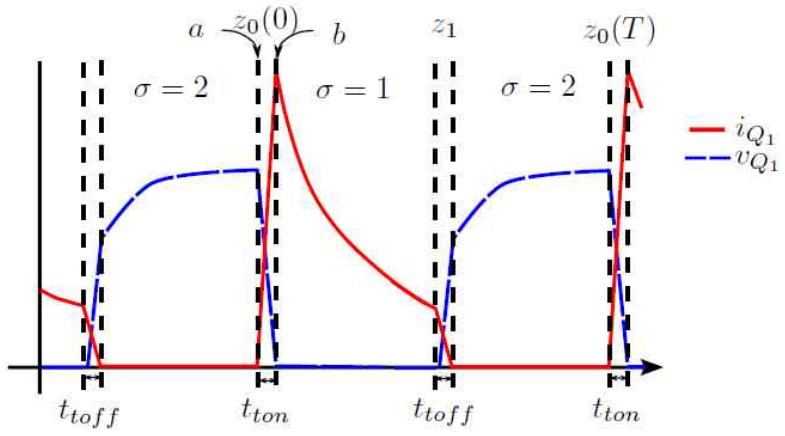

Fig. 6. Switching waveforms of $Q_{1}$ with switch turn-on time $t_{\text {ton }}$ and turn-off time $t_{\text {off }}$.

$$
i_{Q^{*}}^{c b s e}=\mathcal{S}^{*} z_{0}(T k) .
$$

Similar to the case of the GECM, the matrices of Eq. (31) can be decomposed to be used with the original discrete state space vector $x_{0}$ and the input $u$, as follows:

$\mathcal{A}_{\delta}=\left[\begin{array}{cc}A_{\delta} & B_{\delta} \\ 0 & I\end{array}\right], \mathcal{H}^{*}=\left[\begin{array}{ll}H^{*} & J^{*}\end{array}\right], \mathcal{S}^{*}=\left[\begin{array}{ll}S^{*} & Z^{*}\end{array}\right]$.

To compute the switching losses in steady state, the steady-state value of the outputs is required. The steady-state values of the voltages and currents can be computed as follows:

$$
\begin{aligned}
& \left\langle v_{Q^{*}}^{\text {open }}\right\rangle^{s s}=\left(H^{*}\left(I-A_{\delta}\right)^{-1} B_{\delta}+J^{*}\right) u, \\
& \left\langle i_{Q^{*}}^{\text {cbs }}\right\rangle^{s s}=\left(S^{*}\left(I-A_{\delta}\right)^{-1} B_{\delta}+Z^{*}\right) u .
\end{aligned}
$$

Finally, the switching losses in steady state can be obtained using the previous results as follows:

$$
P_{S b s s}=\left(\left\langle i_{Q^{*}}^{\text {dse }}\right\rangle^{s s}\right)^{T} M\left\langle v_{Q^{*}}^{\text {open }}\right\rangle^{s s},
$$

where:

$$
M=\frac{1}{6 T} \operatorname{dag}\left(\rho\left(Q_{1}, 1\right), \ldots, \rho\left(Q_{j}, 1\right), \rho\left(Q_{1}, 2\right), \ldots, \rho\left(Q_{j}, 2\right)\right)
$$

and

$\rho\left(Q_{x}, \gamma\right)=\left\{\begin{array}{ll}t_{\text {ton }} & \text { If } Q_{x} \text { is ON when } \sigma=\gamma \\ t_{\text {toff }} & -\end{array}\right.$,

where the function $d \dot{a} g(*)$ generates a diagonal matrix with the elements of the argument. Matrix $M$ is scaled by $1 / 6$ because of the trapezoidal resistive switching approximation [37].

\section{B. Basic Cell Example}

In this subsection, a numerical example on how to compute the switching losses is applied to the basic ladder multilevel converter shown in Fig. 3. The switches are numbered from bottom to top (Fig. 3). Only the computation of the losses for switches $Q_{1}$ and $Q_{2}$ is shown. Therefore, the following vectors can be defined:

$$
\begin{array}{ll}
v_{Q}^{\text {open }} & =\left[v_{Q_{1}}^{\text {open }}, v_{Q_{2}}^{\text {open }}\right]^{T}, \\
i_{Q}^{\text {cbse }} & =\left[i_{Q_{1}}^{\text {cbse }}, i_{Q_{2}}^{\text {cbse }}\right]^{T} .
\end{array}
$$

1) Compute the Voltages and Currents of the Switches: We compute the matrices $\mathcal{H}$ and $\mathcal{S}$ from Eq. (27). For instance, the calculation of the open voltage of $Q_{1}$ should be computed in the mode $\sigma=2$ (Fig. 4(b)) and $Q_{2}$ should be 
computed in the mode $\sigma=1$ (Fig. 4(a)), as follows:

$$
\begin{aligned}
\mathcal{H} & =\left[\begin{array}{lll}
-\varpi\left(R_{C}+R_{\text {bad }}\right) R_{O N} & \varpi R_{\text {bad }} R_{O N} & 1-\varpi R_{C} R_{O N} \\
\frac{R_{O N}}{\left(2 R_{O N}+R_{C}\right)} & 0 & 1+\frac{R_{O N}}{\left(R_{C}+R_{\text {bad }}\right)}
\end{array}\right], \\
\mathcal{S} & =\left[\begin{array}{lll}
\frac{-1}{\left(2 R_{O N}+R_{C}\right)} & 0 & \frac{1}{\left(2 R_{O N}+R_{C}\right)} \\
\varpi\left(R_{C}+R_{\text {bad }}\right) & -\varpi R_{\text {bad }} & \varpi\left(R_{C}+2 R_{O N}\right)
\end{array}\right],
\end{aligned}
$$

where:

$$
\varpi=\frac{1}{R_{C}\left(R_{C}+2\left(R_{\text {bad }}+R_{O N}\right)\right)+2 R_{\text {bad }} R_{O N}} .
$$

Replacing numerical values, the following results are obtained:

$$
\begin{aligned}
\mathcal{H} & =\left[\begin{array}{lll}
-0.4993 & 0.4993 & 1 \\
0.4997 & 0 & 0.5003
\end{array}\right], \\
\mathcal{S} & =\left[\begin{array}{lll}
-0.2776 & 0 & 0.2776 \\
0.2774 & -0.2774 & 0.43 \times 10^{-6}
\end{array}\right] .
\end{aligned}
$$

2) Create the Matrices $\mathcal{H}^{*}$ and $\mathcal{S}^{*}$ : We expand the matrices $\mathcal{H}$ and $\mathcal{S}$ using Eq. (9), as follows:

$$
\begin{aligned}
\mathcal{H}^{*} & =\left[\begin{array}{lll}
\mathcal{H}^{T} & \left(\mathcal{H} \phi_{1}(d T)\right)^{T}
\end{array}\right]^{T} \\
& \approx\left[\begin{array}{lll}
-0.4993 & 0.4993 & 1 \\
0.4997 & 0 & 0.5003 \\
-0.1414 & 0.4979 & 0.6407 \\
0.1415 & 0 & 0.8585
\end{array}\right], \\
\mathcal{S}^{*} & =\left[\begin{array}{lll}
\mathcal{S}^{T}\left(\mathcal{S} \phi_{1}(d T)\right)^{T}
\end{array}\right]^{T} \\
& \approx\left[\begin{array}{lll}
-0.2776 & 0 & 0.2776 \\
0.2774 & -0.2774 & 0.43 \times 10^{-6} \\
-0.0786 & 0 & 0.0786 \\
0.0785 & -0.2766 & 0.1996
\end{array}\right] .
\end{aligned}
$$

3) Create Matrix $M$ : The matrix $M$ contains the information on the turn-on and turn-off time of the switches. For this numerical example, $t_{\text {ton }}=200 \mathrm{~ns}$ and $t_{\text {toff }}=150 \mathrm{~ns}$.

$$
\begin{gathered}
M=\frac{1}{6 T} \operatorname{dan}\left(\rho\left(Q_{1}, 1\right), \rho\left(Q_{2}, 1\right), \rho\left(Q_{1}, 2\right), \rho\left(Q_{2}, 2\right)\right) \\
=\frac{1}{6 T}\left[\begin{array}{llll}
t_{\text {ton }} & 0 & 0 & 0 \\
0 & t_{\text {toff }} & 0 & 0 \\
0 & 0 & t_{\text {toff }} & 0 \\
0 & 0 & 0 & t_{\text {ton }}
\end{array}\right] \\
M=\left[\begin{array}{llll}
1.7 & 0 & 0 & 0 \\
0 & 1.2 & 0 & 0 \\
0 & 0 & 1.2 & 0 \\
0 & 0 & 0 & 1.7
\end{array}\right] \times 10^{-3}
\end{gathered}
$$

4) Decompose the Matrices: We obtain the matrices from Eq. (32) using the results in Eqs. (10), (43), and (44), as follows:

$$
\begin{array}{rll}
A_{\delta} & =\left[\begin{array}{ll}
0.1529 & 0.4579 \\
0.13 & 0.5373
\end{array}\right], & B_{\delta}=\left[\begin{array}{l}
0.3848 \\
0.3257
\end{array}\right] \\
H^{*} & =\left[\begin{array}{ll}
-0.4993 & 0.4993 \\
0.4997 & 0 \\
-0.1414 & 0.4979 \\
0.1415 & 0
\end{array}\right], & J^{*}=\left[\begin{array}{l}
1 \\
0.5003 \\
0.6407 \\
0.8585
\end{array}\right] \\
S^{*} & =\left[\begin{array}{ll}
-0.2776 & 0 \\
0.2774 & -0.2774 \\
-0.0786 & 0 \\
0.0785 & -0.2766
\end{array}\right] \Omega^{-1}, & Z^{*}=\left[\begin{array}{l}
0.2776 \\
0.43 \times 10^{-6} \\
0.0786 \\
0.1996
\end{array}\right]
\end{array}
$$

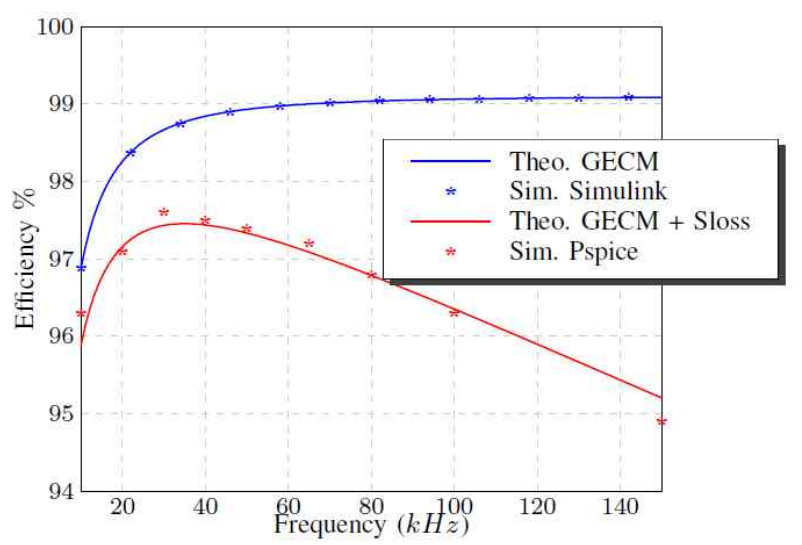

Fig. 7. Comparison of theoretical efficiency with simulation for the basic cell.

5) Compute the Steady-State Values of the Currents and Voltages: We compute the steady-state values of the voltages and currents using Eq. (33) and the results of the previous step $\left(u=V_{\boldsymbol{i}}=340\right)$, as follows:

$$
\left\langle v_{Q^{*}}^{\text {open }}\right\rangle^{s S}=\left[\begin{array}{l}
339.37 \\
337.34 \\
336.51 \\
339.25
\end{array}\right] V,\left\langle i_{Q^{*}}^{\text {cbse }}\right\rangle^{s S}=\left[\begin{array}{l}
1.48 \\
0.349 \\
0.419 \\
1.939
\end{array}\right] A .
$$

6) Compute the Switching Power Losses:

Using Eqs. (34) and (45) and the results of the previous step, the total switching power losses for $Q_{1}$ and $Q_{2}$ can be computed as follows:

$$
P_{\text {Sbss }}=\left(\left\langle i_{Q^{*}}^{\text {dss }}\right\rangle^{s S}\right)^{T} M\left\langle v_{Q^{*}}^{\text {open }}\right\rangle^{s s}=2.26 \mathrm{~W} .
$$

With consideration of the switching power losses, the efficiency of the converter can be computed as follows:

$$
E_{f f}=\frac{P_{\text {out }}}{P_{\text {out }}+P_{\text {Cbss }}+P_{\text {Sbss }}} \text {. }
$$

For this case, the output voltage of the converter is not significantly affected by switching losses. Therefore, $P_{\text {out }}$ and $P_{\text {Cbss }}$ can be computed with the GECM. Fig. 7 shows a comparison between the theoretical calculation of efficiency (with and without switching losses) and the respective simulations for different switching frequencies while considering all switches in the basic cell. The estimation obtained using the analytical method reflects correctly the behavior of these losses.

\section{ANALYSIS OF THE CONVERTER}

In this section, the DLT is analyzed using the new techniques introduced in Sections III and IV. An in-depth analysis is conducted for this topology to show the effect on the efficiency caused by the switching frequency and the parasitic resistances of the switches. The GECM is validated using the DLT in [36].

Fig. 8 shows an equivalent model of the converter. In this model, $N$ represents the ideal voltage gain and the resistance 


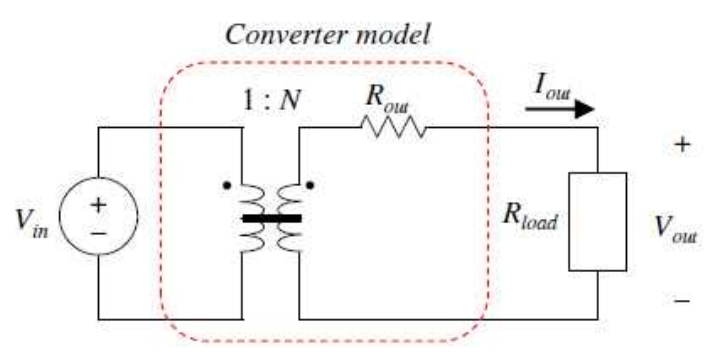

Fig. 8. Equivalent model of an SC converter with a DC/DC transformer, where $N$ is the gain of the converter with no load and $R_{\text {out }}$ is the output resistance.

$R_{\text {out }}$ represents the losses. The resistance $R_{\text {out }}$ is dependent on the switching frequency of the converter and the values of ON resistances of the switches and ESR of the capacitors.

Given that the new modeling technique is able to model accurately the SC converter, it is used to analyze and optimize the DLT for implementation.

The nominal values of the converter are as follows: input voltage $V_{\dot{n}}=350 \mathrm{~V}$; output voltage $V_{\text {out }}=3,000 \mathrm{~V}$; output current $I_{\text {out }}=100 \mathrm{~mA}$; number of cells $N_{c}=8$. For this example, a commercial capacitor $C=2.2 \mu \mathrm{F}, E S R=$ $2.5 \mathrm{~m} \Omega$ is used.

Using the GECM, we observed that the duty cycle of maximum gain is independent of the load value and switching frequency and is always $d=0.5$. Moreover, the gain $V_{\text {out }} / d$ is insufficient to use $d$ as control signal. Therefore, the duty cycle is set to a constant value $d=0.5$.

As mentioned previously, no conclusion on efficiency can be easily drawn because of the effect on the current waveform when the time constants are small compared with the switching period. Using the results obtained in Section IV, the efficiency of the converter can be computed analytically.

The following criteria is considered when selecting an adequate value for the switching frequency and $R_{S}$ : The value of $R_{S}$ should be as high as possible without compromising efficiency. In this converter, the lower the $R_{S}$, the higher the current spikes in the switches, producing overcurrents.

Fig. 9 shows efficiency as a function of $f$ and $R_{S}$. The values of $R_{S}$ and $f$ are selected as follows: First, the highest possible value of $R_{S}$ that can be classified into the lighter region is selected and restricted to commercial values. A frequency value that can also be classified into this region is then selected. Fig. 10 shows the detailed effect of switching frequency on a given $R_{S}$. The value that produces the highest efficiency can be easily selected. The values of $R_{S}=1.8 \Omega$ (N-MOS transistor P8NK100Z) and $f=80 \mathrm{kHz}$ are selected, with an efficiency of $92.5 \%$. We observed no increments in efficiency of over $1 \%$ for any other possible values of frequency and resistance.

Fig. 10 shows the efficiency of three different switch

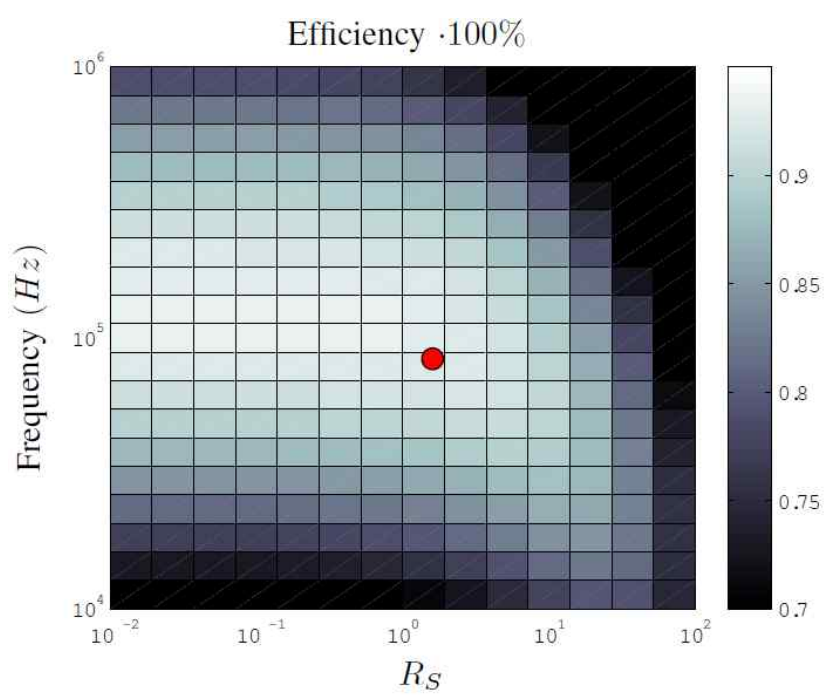

Fig. 9. Efficiency as a function of the switch resistance $R_{S}$ and switching frequency $f$. The dot marks the selected values $R_{S}=1.8 \Omega$ and $f=80 \mathrm{kHz}$, with an efficiency of $92.5 \%$.

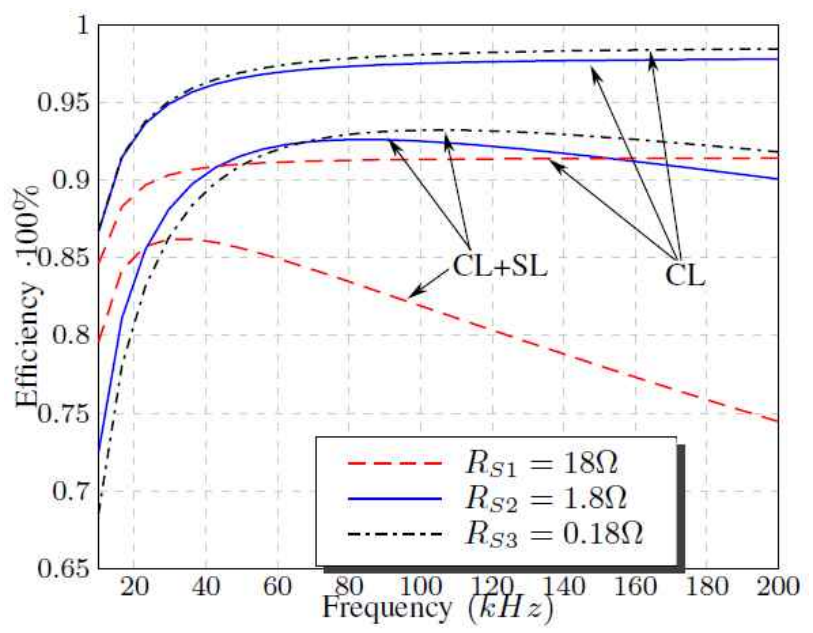

Fig. 10. Comparison of the efficiency obtained using the theoretical model for three different values of switch ON resistance $R_{S}$ (conduction losses only $(\mathrm{CL})$ and conduction and switching losses $(\mathrm{CL}+\mathrm{SL})$ ).

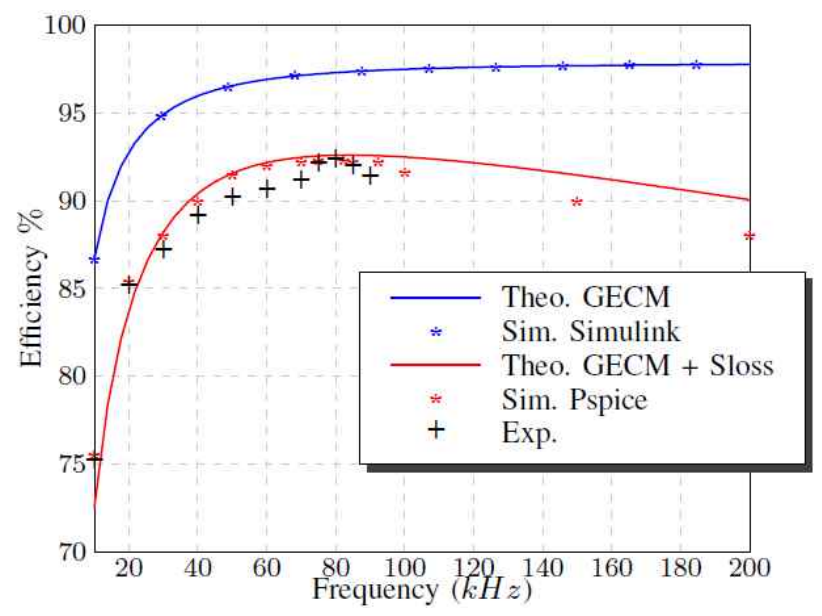

Fig. 11. Comparison of the efficiency obtained using the theoretical model with and without switching losses, simulation results, and experimental measurements with $R_{S}=1.8 \Omega$. 
resistances as a function of switching frequency to compare in detail the influence of switching frequency and switch resistance. The results of the GECM (where no switching losses are considered) are compared with the results of the GECM with the addition of switching losses for each switch. When the charging and discharging process of capacitors is completed in one switching period, the losses are independent of the $R_{S}$ (conduction only) value and are attributed to the inherent energy losses caused by voltage differences of the capacitors when they are connected. However, including switching losses, at low frequencies, the lower the $R_{S}$, the higher the losses.

Although efficiency is slightly higher with $R_{S}=0.18 \Omega$ at different frequencies in comparison with $R_{S}=1.8 \Omega$ at $f=80 \mathrm{kHz}$ (increment $<1 \%$ ), the current spikes are dramatically higher with $R_{S}=0.18 \Omega$ (up to $16.6 \mathrm{~A}$ ) in comparison with the selected values (up to $3.3 \mathrm{~A}$ ).

Experimental measurements are conducted to validate the analysis of the converter. Fig. 11 shows a comparison among the analytical, simulated, and experimental efficiencies. The simulated and experimental results present a relative error below 4\% compared with the analytical results, with switching losses for all the tested cases. Power loss attributed to switching is $5.5 \%$, with $R_{S}=1.8 \Omega$ and $f=80 \mathrm{kHz}$.

\section{CONCLUSIONS}

In this study, a method for computing switching losses is introduced, and a new modeling technique is used to analyze the efficiency of an SC converter. This technique is helpful when the dynamics of each mode of the switched system are characterized as rapid when compared with the switching period.

The DLT was analyzed with the use of the new model, and the optimal parameter values were obtained. We observed that the highest efficiency is not necessarily achieved with the smallest resistor values and it depends on the switching frequency. Therefore, careful analysis is required to achieve the highest efficiency point.

Experimental measurements and simulations were conducted to validate the analytical results. An efficiency of $92.5 \%$ was obtained at $80 \mathrm{kHz}$, with switching losses of $5.5 \%$.

\section{ACKNOWLEDGMENT}

The authors acknowledge the support provided by the research project "Conversión de múltiples puertos, interconexión y asignación dinámica de energía de generadores PV distribuidos" of Pontificia Universidad Javeriana-Vicerectoría de Investigación-Project No. 004018

\section{REFERENCES}

[1] J. M. Henry and J. W. Kimball, "Practical performance analysis of complex switched-capacitor converters," IEEE Trans. Power Electronics, Vol 26, No. 1, pp. 127-136, Jun. 2011.

[2] K. Sano and H. Fujita, "Performance of a high-efficiency switched-capacitor-based resonant converter with phase-shift control," IEEE Trans. Power Electron., Vol. 26, No. 2, pp. 344-354, Feb. 2011

[3] A. Elasser, M. H. Kheraluwala, M. Ghezzo, R. L. Steigerwald, N. A. Evers, J. Kretchmer, and T. P. Chow, "A comparative evaluation of new silicon carbide diodes and state-of-the-art silicon diodes for power electronic applications," IEEE Trans. Ind. Appl., Vol. 39, No. 4, pp. 915-921, Jul./Aug. 2003.

[4] J. Ebrahimi, E. Babaei, and G. B. Gharehpetian, "A new topology of cascaded multilevel converters with reduced number of components for high-voltage applications," IEEE Trans. Power Electron., Vol. 26, No. 11, pp. 3109-3118, Nov. 2011.

[5] J.S. Lai and F. Zheng Peng, "Multilevel converters-a new breed of power converters," IEEE Trans. Ind. Appl., Vol. 32, No. 3, pp. 509-517, May/Jun. 1996.

[6] J. Rodriguez, J. S. Lai, and F. Z. Peng, "Multilevel inverters: A survey of topologies, controls, and applications," IEEE Trans. Ind. Electron., Vol. 49, No. 4, pp. 724-738, Aug. 2002.

[7] J. Wang, B. Wu, D. Xu, and N. R. Zargari, "Multimodular matrix converters with sinusoidal input and output waveforms," IEEE Trans. Ind. Electron., Vol. 59, No. 1, pp. 17-26, Jan. 2012.

[8] J. Zhao, Y. Han, X. He, C. Tan, J. Cheng, and R. Zhao, "Multilevel circuit topologies based on the switched-capacitor converter and diode-clamped converter," IEEE Trans. Power Electron., Vol. 26, No. 8, pp. 2127-2136, Aug. 2011.

[9] B. Cougo, G. Gateau, T. Meynard, M. Bobrowska-Rafal, and M. Cousineau, "PD modulation scheme for three-phase parallel multilevel inverters," IEEE Trans. Ind. Electron., Vol. 59, No. 2, pp. 690-700, Feb. 2012.

[10] J. Ebrahimi, E. Babaei, and G. B. Gharehpetian, "A new multilevel converter topology with reduced number of power electronic component," IEEE Trans. Ind. Electron., Vol. 59, No. 2, pp. 655-667, Feb. 2012.

[11] Y. Hinago and H. Koizumi, "A switched-capacitor inverter using series/parallel conversion with inductive load," IEEE Trans. Ind. Electron., Vol 59, No. 2, pp. 878-887, Feb. 2012.

[12] Y. P. Hsieh, J. F. Chen, T. J. Liang, and L. S. Yang, "Novel high step-up DC-DC converter with coupled-inductor and switched-capacitor techniques," IEEE Trans. Ind. Electron., Vol. 59, No. 2, pp. 998-1007, Feb. 2012

[13] A. Ioinovici, "Switched-capacitor power electronics circuits," IEEE Circuits Syst. Mag., Vol. 1, No. 3, pp. 37-42, Jul. 2001.

[14] K. Jin, M. Xu, and F. C. Lee, "A switching-capacitor PWM DC-DC converter and its variations," IEEE Trans. Power Electron., Vol. 25, No. 1, pp. 24-32, Jan. 2010.

[15] M. Khazraei, H. Sepahvand, K. A. Corzine, and M. Ferdowsi, "Active capacitor voltage balancing in single-phase flying-capacitor multilevel power converters," IEEE Trans. Ind. Electron., Vol. 59, No. 2, pp.769-778, Feb. 2012.

[16] A. Lopez, R. Diez, G. Perilla, and D. Patino, "Analysis and comparison of three topologies of the ladder multilevel dc/dc converter," IEEE Trans. Power Electron., 
Vol. 27, No. 7, pp. 3119-3127, Jul. 2012.

[17] A. Lopez, D. Patino, R. Diez, and G. Perilla, "An equivalent continuous model for switched systems," Systems \& Control Letters, Vol. 62, No. 2, pp. 124-131, Feb. 2013.

[18] S. Ben-Yaakov, "On the influence of switch resistances on switched-capacitor converter losses," IEEE Trans. Ind. Electron., Vol. 59, No. 1, pp. 638-640, Jan. 2012.

[19] M. D. Seeman and S. R. Sanders, "Analysis and optimization of switched-capacitor DC-DC converters," IEEE Trans. Power Electron., Vol. 23, No. 2, pp. 841-851, Mar. 2008.

[20] C. K. Tse, S. C. Wong, and M. H. L. Chow, "On lossless switched-capacitor power converters," IEEE Trans. Power Electron., Vol. 10, No. 3, pp. 286-291, May 1995.

[21] A. Lopez, R. Diez, G. Perilla, and D. Patino, "Convertidor multinivel DC/DC de alto voltaje en topologia escalera," Ingenieria y Universidad, Pontificia Universidad Javeriana, Vol. 16, No. 1, pp. 117-133, Oct. 2012.

[22] F. Canales, P. M. Barbosa, J. M. Burdio, and F. C. Lee, "A zero voltage switching three-level $\mathrm{DC} / \mathrm{dc}$ converter," in Proc. INTELEC Telecommunications Energy Conf. Twenty-second Int, pp. 512-517, 2000.

[23] D. Cao and F. Zheng Peng, "A family of zero current switching switched-capacitor dc-dc converters," in Proc. Twenty-Fifth Annual IEEE Applied Power Electronics Conf. and Exposition (APEC), pp. 1365-1372, 2010.

[24] C. Chakraborty, M. Ishida, and Y. Hori "Novel resonant converter topology realized by adjusting transformer parameters," in Proc. 26th Annual Confjerence of the IEEE Industrial Electronics Society IECON 2000, Vol. 1, pp. 320-325, 2000.

[25] M. L. Gebben, J. G. Cintron-Rivera, W. Qian, D. Cao, X. Pei, and F. Zheng Peng, "A zero-current-switching multilevel switched capacitor dc-dc converter," in Proc. IEEE Energy Conversion Congress and Exposition (ECCE), pp. 1291-1295, 2011.

[26] O. Keiser, P. K. Steimer, and J. W. Kolar, "High power resonant switched-capacitor step-down converter," in Proc. IEEE Power Electronics Specialists Conf. PESC, pp. 2772-2777, 2008.

[27] Y. S. Kim, B. S. Seo, and D. S. Hyun, "A novel structure of multi-level high voltage source inverter," in Proc. Computer, Communication Control and Power Engineering IEEE Region 10 Conf TENCON, No. 0, pp. 503-508, 1993.

[28] T. A. Meynard and H. Foch., "Multi-level conversion: high voltage choppers and voltage-source inverters," in Proc. Annual IEEE Power Electronics Specialists Conf. PESC, pp. 397-403, 1992.

[29] K. Y. Lee and Y.S. Lai, "A novel magnetic-less bi-directional dc-dc converter," in Proc. 30th Annual Conf. of IEEE Industrial Electronics Society IECON, Vol. 2, pp. 1014-1017, 2004.

[30] O. C. Mak and A. Ioinovici, "Small size and low weight $\mathrm{dc} / \mathrm{dc}$ converter with no magnetic elements," in Proc. Of Int. Telecommunications Energy Conf. INTELEC, pp. 573-580, 1994.

[31] G. Zhu and A. Ioinovici, "Switched-capacitor power supplies: DC voltage ratio, efficiency, ripple, regulation," in Proc. IEEE Int Circuits and Systems ISCA., 'Connecting the World'. Symp, Vol. 1, pp. 553-556, 1996.

[32] R.D. Middlebrook and S. Cuk, "A general unified approach to modelling switching-converter power stages," in Proc. IEEE Power Electronics Specialist Conference, pp. 73-86, 1976

[33] M. N. Gitau, C. K. Konga, and J. G. V. D. Westhuizen, "Loss reduction in generalised multilevel converters," in Proc. AFRICON '09, pp. 1-6, 2009.

[34] C. K. Tse, S. C. Wong, and M. H. L. Chow, "The simplest lossless switched-capacitor ac/dc converter," in Proc. Annual IEEE Power Electronics Specialists Conf., PESC, pp. 1212-1217, 1994.

[35] A. Lopez, D. Patino, R. Diez, and G. Perilla, "A DC/DC multilevel converter for high voltage applications," in Proc. IEEE IX Latin American and IEEE Colombian Conf Robotics Symp. Automatic Control and Industry Applications (LARC), pp. 1-5, 2011.

[36] A. Lopez, D. Patino, and R. Diez, "Validation of generalized continuous equivalent model on a DC/DC ladder multilevel converter," in Proc. IEEE Energy Conversion Congress and Exposition (ECCE), pp. 4281-4287, 2012.

[37] R. W. Erickson and D. Maksimovic, Fundamentals of Power Electronics, 2nd ed, Kluwer Academic Publishers, 2001.

[38] Ned Mohan, Tore M. Undeland, and William P. Robbins. Power Electronics, Converters, Applications, and Design, 2nd ed., John Wiley \& Sons, 1995.

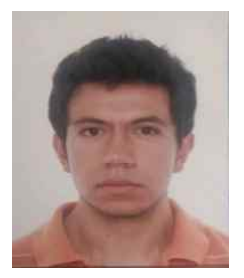

Andrés Lopez obtained his B.Sc. degree in Electronics Engineering from Pontificia Universidad Javeriana, Bogotá, Colombia in 2010. He obtained his M.Sc. degree from the same university in 2012. Currently, he is doing his Ph.D. in Australia. His research interests include SC converters and modeling and control of power converters.

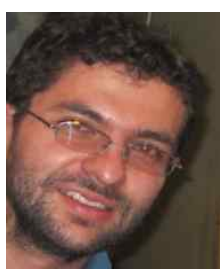

Diego Patino was born in Manizales Colombia He has written several articles. He obtained his degree in Electronics Engineering from la Universidad Nacional. He obtained his Master's Degree from la Universidad de Los Andes, Bogota. He has a Ph.D. in automatic control from Nancy University, France. Currently, he works as full-time professor at Pontificia Universidad Javeriana, Bogota, Colombia. His main research interests are hybrid dynamical systems, power converters, and nonlinear control theory.

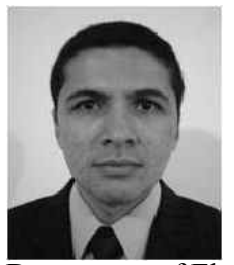

Rafael Diez received his B.Sc. degree in Electronics Engineering from Pontificia Universidad Javeriana, Bogotá, Colombia in 2001. He obtained his M.Sc. in Microelectronics in 2005 and his Ph.D. in Electrical Engineering in 2008 from Université de Toulouse, France. He is currently with the Department of Electronics of Pontificia Universidad Javeriana. His main interest is the development of power converters for electric discharges. 